\title{
Dielectrophoretic Tweezers for Pickup and Relocation of Individual Cells Using Microdisk Electrodes with a Microcavity Taishu TANAKA, Fumio MIZUTANI, and Tomoyuki YASUKAWA*
}

Graduate School of Material Science, University of Hyogo, 3-2-1 Kouto, Kamigori, Ako, Hyogo 678-1297, Japan

* Corresponding author: yasu@sci.u-hyogo.ac.jp

\begin{abstract}
We describe here the dielectrophoretic manipulation using a microdisk electrode with microcavity for picking up, positioning and relocating single target cells. The attractive force of positive dielectrophoresis ( $p$-DEP) was used to trap target cells at the electrode tip, while trapped cells were released by the repulsive force of negative DEP ( $n$-DEP). The capturing of cells in the cavity allows them to transfer at the desired position even after turning off the attractive force by $p$-DEP. We demonstrate an application of the present technique in the patterning with living cells.
\end{abstract}

(C) The Electrochemical Society of Japan, All rights reserved.

Keywords : Dielectrophoresis, Tweezers, Microdisk Electrode, Single Cell Manipulation

\section{Introduction}

Dielectrophoresis (DEP) is an attractive technique for manipulating cells by imposing alternating current (AC) electric field gradients and is applied to form patterns with cells. In many cases, a microelectrode array fabricated on a solid substrate was used to form the heterogeneous electric field for large-scale manipulation of cells. $^{1-3}$ Although the handling of single cells is important for investigating characteristics of individual cells, this technique is not suitable to pick the target cells up from the random cell population and relocate them at the desired position. Optical tweezers enable single cell capturing with excellent accuracy, ${ }^{4,5}$ but require relatively large and expensive systems for the manipulation.

DEP tweezers consisted of the needle type of microelectrodes have been reported to manipulate single target cells. ${ }^{6-10} \mathrm{AC}$ electric field was formed by applying voltage between the microelectrode tip and ground electrode. The attractive force of positive DEP (p-DEP) was used to trap a target cell at the tip. After the trapped cells were transferred to the desired position by a mechanical micromanipulator, they were released by the repulsive force of negative DEP (n-DEP). However, the DEP force must be continuously applied during a cell transfer, because the trapped cells were released from the electrode tip by turning off the voltage. The cell damage should arise by exposing the cell to the strong electric field formed at the tip for a long period. In addition, the microelectrode with the conductive side which was not insulated from the solution gave rise to the capture of the several cells at the single tip. Previously, we reported the manipulation of cells with a dual-microdisk electrode which consisted of two microdisk electrodes in a glass capillary. ${ }^{10}$ The fabrication of dual-electrodes of equal value is difficult due to the necessity of the insertion of two metal wires in the single capillary.

In the present study, we have used a microdisk electrode with the microcavity as a container for a single target cell. We demonstrate the trapping of target cells in the cavity by p-DEP, positioning at the desired position without the application of attractive force and releasing of trapped cells by n-DEP, and apply them to form patterns with living cells.

\section{Experimental Section}

The needle type of Pt microelectrode tip with $20 \mu \mathrm{m}$ diameter was fabricated. The AC voltage $\left(10 \mathrm{~V}_{\mathrm{pp}}, 200 \mathrm{~Hz}\right)$ was applied to the tip which is immersed in the saturated $\mathrm{NaNO}_{3}$ solution during $10 \mathrm{~s}$ to form the microcavity at the top of the tip by an electrolytic etching. The etched depth was approximately $10 \mu \mathrm{m}$. The tip was mounted on an xyz micromanipulator (Suruga Seiki ltd., Tokyo). The outer wall at the tip was contacted to an ITO substrate with $14^{\circ}$ of angle relatively to the ITO substrate.

PAI murine myeloma cells ${ }^{11}$ were grown in complete RPMI 1640 medium supplemented with $10 \%$ fetal bovine serum, 50 units $\mathrm{mL}^{-1}$ penicillin, and $50 \mu \mathrm{g} \mathrm{mL}^{-1}$ streptomycin, at $37^{\circ} \mathrm{C}$ under $5 \% \mathrm{CO}_{2}$. Myeloma cells are spherical cell having a diameter in the range of $10-15 \mu \mathrm{m}$. The solution used was a $250 \mathrm{mM}$ sucrose solution with the conductivity of $0.16 \mathrm{mS} \mathrm{m}^{-1}$. The tip was placed close to the target cell on the ITO substrate. The AC voltage with the frequency for p-DEP $(10 \mathrm{MHz})$ was then applied to the tip to capture the cell in the microcavity with a function generator (wave factory WF1945, NF Co., Japan), while the ITO was connected to the ground. The voltage was immediately turned off after trapping the cell. The captured cell was transferred to the desired position by moving the tip and was released from the cavity by the repulsive force of n-DEP $(1.0 \mathrm{kHz})$. The arrangement of cells were obtained by repeating a series of procedures for each cell.

The esterase activities in the living myeloma cells were investigated by staining the cells with 5-(and -6)-carboxyfluorescein diacetate, succinimidyl ester (CFDA SE, Thermo Fisher Scientific K.K.). CFDA SE is hydrolyzed to carboxyfluorescein, succinimidyl ester (CFSE) which shows the green fluorescence in the living cells by the enzyme reaction of esterase presented in the cytoplasm. After the cells were trapped in the microcavity by p-DEP or released from the microcavity by n-DEP, the solution of $1.2 \mu \mathrm{M}$ CFDA SE was added in sucrose solution. Then, green fluorescence signals were observed after $10 \mathrm{~min}$.

\section{Results and Discussion}

The capture of cells by p-DEP was investigated. Figure 1 shows a series of images for capturing a cell in the cavity at the electrode tip. Cells were dispersed in $250 \mathrm{mM}$ sucrose. The surface of the electrode was positioned at $50 \mu \mathrm{m}$ right from the center of the target cell (Fig. 1a). When $A C$ voltage of $10 \mathrm{~V}_{\mathrm{pp}}$ with the frequency of $10 \mathrm{MHz}$ was applied, the cell with diameter of $12 \mu \mathrm{m}$ began to direct toward the tip (Figs. 1b-1d) and moved to $45 \mu \mathrm{m}$ right to capture in the macrocavity at $16 \mathrm{~s}$ (Fig. 1e). Relatively high electric field was formed in the microcavity due to the configuration between the microelectrode and ITO substrate with a large conductive surface. 

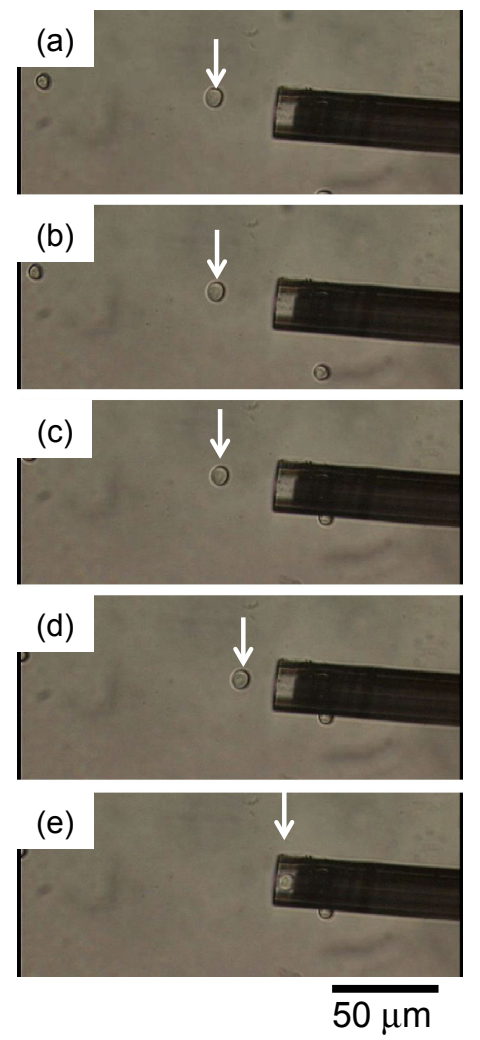

Figure 1. (Color online) Images for capturing a cell in the cavity (a) before and (b) 5, (c) 10, (d) 15, and (e) $16 \mathrm{~s}$ after applying the voltage.

Therefore, the attractive force of p-DEP by the tip with microcavity can be applicable for trapping a single cell.

We investigated the velocity of cell which moves to the tip by p-DEP applied the voltage of $10 \mathrm{~V}_{\mathrm{pp}}$ at the frequency of $10 \mathrm{MHz}$. The velocity during the cell capturing dramatically increased with decreasing the distance between the cell and the tip. The average velocity directly before the cell was trapped reached at ca. $600 \mu \mathrm{m} \mathrm{s}^{-1}$ due to the shift of $20 \mu \mathrm{m}$ per frame (30 frames per second). The increase of the velocity is attributed to the increase of the electric field by decreasing the distance. When the cell moves in the solution, the dielectrophoretic force is approximately equal to the viscosity resistance of the solution. Therefore, the force operating on a cell can be estimated by Stokes law $(F=6 \pi a \eta v)$, where a is the radius of the cell $(6.0 \mu \mathrm{m}), \eta$ is the solvent viscosity $(1.0 \times$ $10^{-3} \mathrm{Nm}^{-2} \mathrm{~s}$ ) and $v$ is the velocity of the cell. Thus, the dielectrophoretic forces were $68 \mathrm{pN}$ at $10 \mu \mathrm{m}$ and $1.2 \mathrm{pN}$ at $25 \mu \mathrm{m}$ away from the electrode surface.

We also studied the velocity and dielectrophoretic force of a cell captured by applying AC voltage with $15 \mathrm{~V}_{\mathrm{pp}}$ and $20 \mathrm{~V}_{\mathrm{pp}}$. The dielectrophoretic force which the cell were applied directly before trapping the cell with $15 \mathrm{~V}_{\mathrm{pp}}$ and $20 \mathrm{~V}_{\mathrm{pp}}$ were at ca. $960 \mathrm{pN}$ $\left(850 \mu \mathrm{m} \mathrm{s}^{-1}\right)$ at $15 \mu \mathrm{m}$ left from the electrode surface and $1.2 \mathrm{nN}$ $\left(1100 \mu \mathrm{m} \mathrm{s}^{-1}\right)$ at $18 \mu \mathrm{m}$ left, respectively. The velocity and force increased with increasing the applied voltage due to the increase of the electric field around the tip electrode. The cells within the distance of $65 \mu \mathrm{m}$ can be trapped by applying the voltage of $10 \mathrm{~V}_{\mathrm{pp}}$. However, several cells could not be captured by the attractive force even when $20 \mathrm{~V}_{\mathrm{pp}}$ was applied to the tip positioned close to the cells. The phenomenon could be due to the non-specific adsorption of cells onto the ITO surface at the force above $1.2 \mathrm{nN}$.

The esterase activity in the trapped cells were investigated by staining the cells with CFDA SE. The optical image and the combination of the optical and fluorescence images are shown in
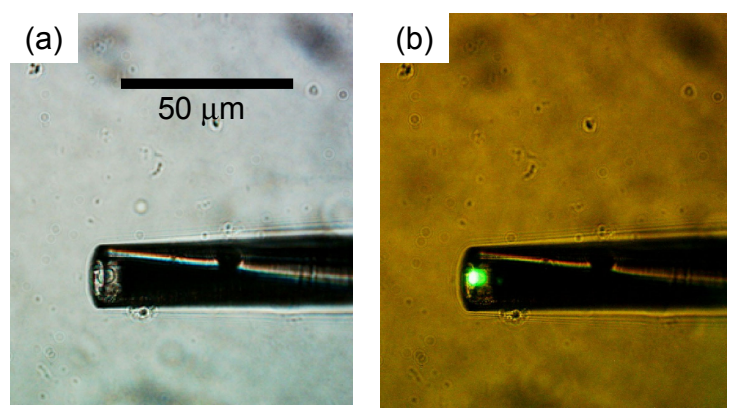

Figure 2. (Color online) (a) Optical image and (b) the combination of the optical and fluorescence images of the cell trapped in the microcavity by $\mathrm{p}$-DEP.
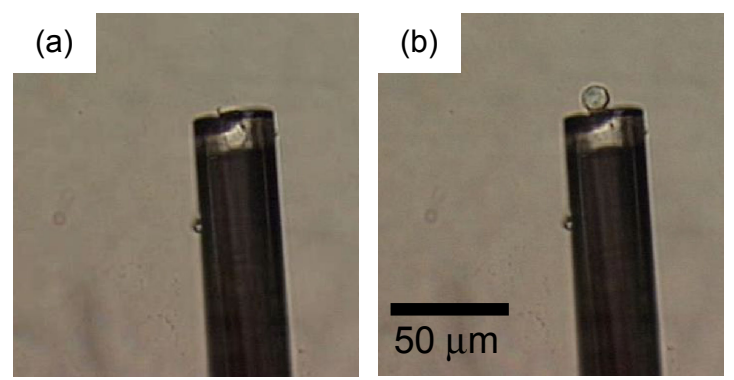

Figure 3. (Color online) Images of the cell (a) trapped in the microcavity by $p$-DEP and (b) released by $n$-DEP.

Figs. $2 \mathrm{a}$ and $2 \mathrm{~b}$. When AC voltage of $20 \mathrm{~V}_{\mathrm{pp}}$ was applied to the microelectrode, the cells positioned $30 \mu \mathrm{m}$ left from the electrode surface was trapped in the cavity with approximately $3 \mathrm{~s}$ (Fig. 2a). The AC voltage was switched off immediately after the cell was trapped. The fluorescence image was obtained $10 \mathrm{~min}$ after adding the solution containing 1.2 $\mu \mathrm{M}$ CFDA SE. The clear green fluorescence signal was observed from the position of the trapped cell in the microcavity (Fig. 2b). The result indicates that the cell trapped in the microcavity by p-DEP maintained the esterase activity and was alive.

To release cells at the desired position, repulsive force of n-DEP was employed in the following experiments. Figure 3 shows the optical images of the cell trapped in the microcavity by p-DEP (Fig. 3a), and the released cell $20 \mathrm{~s}$ after the voltage and frequency were switched to $2.0 \mathrm{~V}_{\mathrm{pp}}$ and $1.0 \mathrm{kHz}$, respectively (Fig. 3b). Again, the target cell directed toward the microcavity by attractive p-DEP force (Fig. 3a). The trapped cell released gradually and positioned at the point of the tip after the voltage and frequency were switched (Fig. 3b). The repulsive force caused by n-DEP directed the cell in microcavity toward the outside of the microcavity. The fluorescence signals of CFSE generated by the cellular esterase reaction were also observed from the cells released into the solution by n-DEP. However, when the high voltage (e.g. $10 \mathrm{~V}_{\mathrm{pp}}$ ) was applied to release the cell, the trapped cell was suddenly out and reached at several tens micrometer far from the tip. The trapped cell remained in the microcavity after the voltage was turned off. Therefore, we used the $\mathrm{n}$-DEP force formed by applying the voltage of $2.0 \mathrm{~V}_{\mathrm{pp}}$ to arrange the cells at the desired position precisely.

The patterning with cells was performed by the capture and release of individual cells with p-and n-DEP. Figure 4a depicts the images of the patterned cells. Cell (a) was trapped in the microcavity by $\mathrm{p}-\mathrm{DEP}$ at $10 \mathrm{~V}_{\mathrm{pp}}$ and $10 \mathrm{MHz}$. The microcavity with trapped cell (a) was moved to the upper left in Fig. 4a at $100 \mu \mathrm{m} \mathrm{s}^{-1}$ after turning off the voltage. The trapped cell has been remained in the microcavity during the transfer to the desired position because the 

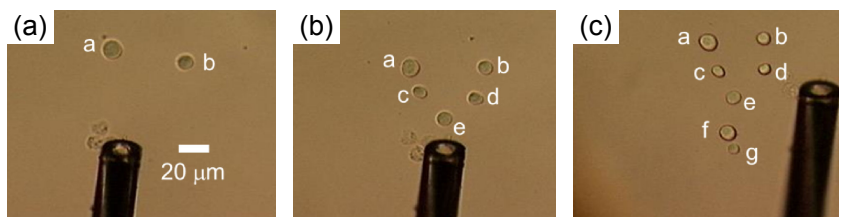

Figure 4. (Color online) Images of cells patterned with (a) 2 , (b) 5, and (c) 7 cells.

microcavity would prevent the releasing of the cell into the solution by the viscosity resistance formed by moving the tip. After manipulation to the desired position, the trapped cell was then released from the microcavity by $\mathrm{n}-\mathrm{DEP}$ at $2.0 \mathrm{~V}_{\mathrm{pp}}$ and $1.0 \mathrm{kHz}$, and positioned at the precise address. The procedure of capture, transfer and release of the cell was repeated for other 6 cells (b)-(g), resulting in the pattern with the alphabet "Y" was formed with 7 cells (Figs. 4b and 4c). In forming the alphabet "Y" with 7 cells, no cell was released from the microcavity during the transfer to the desired position even after the attractive force of p-DEP was switched off. However, the use of the microelectrode without the microcavity led to the release of cells trapped at the electrode surface even though cells were directed to the electrode by the attractive force of $p$-DEP. Therefore, the presence of the microcavity is advantageous to transfer the trapped cells to the desired positions.

The time required for the positioning of a single cell is as short as $1 \mathrm{~min}$. For this patterning, we have used the microelectrode with the microcavity. The trapped cells by $\mathrm{p}$-DEP were maintained during the transfer to the desired position. The present system is promising for applications to the restoration of the patterned cells formed by other methods and co-culture of the different types of cells by regulating the distance between the cells.

\section{Acknowledgments}

This study was partly supported by a Grant-in-Aid for Scientific Research on Innovative Areas "Bio Assembler" of The Ministry of Education, Culture, Sports, Science and Technology, Japan.

\section{References}

1. R. Pethig, Biomicrofluidics, 4, 022811 (2010).

2. J. Voldman, Annu. Rev. Biomed. Eng., 8, 425 (2006).

3. H. Hatanaka, T. Yasukawa, and F. Mizutani, Anal. Chem., 83, 7207 (2011).

4. A. Ashkin, J. M. Dziedzic, J. E. Bjorkholm, and S. Chu, Opt. Lett., 11, 288 (1986).

5. D. G. Grier, Nature, 424, 810 (2003).

6. T. Matsue, N. Matsumoto, S. Koike, and I. Uchida, Biochim. Biophys. Acta, 1157, 353 (1993).

7. T. Schnelle, T. Müller, R. Hagedorn, A. Voigt, and G. Fuhr, Biochim. Biophys. Acta, 1428, 99 (1999).

8. K. Lee, S. G. Kwon, S. H. Kim, and Y. K. Kwak, Sens. Actuators, A, 136, 154 (2007).

9. D. M. Graham, M. A. Messerli, and R. Pethig, Biotechniques, 52, 39 (2012)

10. S. Ogata, T. Yasukawa, and T. Matsue, Bioelectrochemistry, 54, 33 (2001).

11. J. W. Stocker, H. K. Forster, V. Miggiano, C. Stahli, G. Staiger, B. Takacs, and T. Staehelin, Res. Disclosure., 217, 155 (1982). 